\title{
Retrospective Investigation of Factors Influencing Time-dependent Changes in Serum Magnesium Levels in Patients Receiving Cetuximab
}

\author{
Kaito Yamashiro \\ Kindai University \\ Atsushi Hirata \\ Kindai University Nara Hospital \\ Ryosuke Ota \\ Kindai University Nara Hospital \\ Fumihiko Ogata \\ Kindai University \\ Takehiro Nakamura \\ Kindai University \\ Naohito Kawasaki ( $\sim$ Kawasaki@phar.kindai.ac.jp ) \\ Kindai University
}

\section{Research Article}

Keywords: cetuximab, time-dependent changes, hypomagnesemia, baseline serum sodium level, magnesium oxide tab, serum magnesium level

Posted Date: April 28th, 2021

DOI: https://doi.org/10.21203/rs.3.rs-443200/v1

License: (9) This work is licensed under a Creative Commons Attribution 4.0 International License. Read Full License 


\section{Abstract}

Background: Cetuximab causes electrolyte abnormalities, such as hypomagnesemia, hypokalemia, and hypocalcemia. However, little is known about the relationships between the onset of hypomagnesemia, patient background before administration, and time-dependent changes in serum magnesium levels. Therefore, we examined the patient backgrounds that influenced the onset of hypomagnesemia and the time-dependent changes in serum magnesium levels in patients receiving cetuximab.

Methods: A retrospective study was performed to investigate patients with advanced or recurrent colorectal cancer or head and neck cancer, treated with a cetuximab regimen from 2012 to 2020 at Kindai University Nara Hospital. In total, 52 patients who met the inclusion criteria were enrolled in this study.

Results: The serum magnesium levels tended to decline with an increasing number of cetuximab administrations. After two administrations of cetuximab, the serum magnesium level was significantly lower in the hypomagnesemia group than in the non-hypomagnesemia group $(p<0.001)$. The multivariate logistic regression analysis revealed that the baseline serum sodium levels (odds ratio: 0.729, 95\% confidence interval: $0.552-0.963$ ) and the combination of magnesium oxide tablet (odds ratio: $0.997,95 \%$ confidence interval: $0.995-0.999$ ) were one of the independent factors for hypomagnesemia. These results indicated that the two administrations of cetuximab may cause magnesium deficiency even if the serum levels are within the normal range. Additionally, hyponatremia before administration may be an indicator of serum magnesium levels after administration of cetuximab.

Conclusion: Cetuximab-induced hypomagnesemia may be predicted using baseline serum sodium levels, and hypomagnesemia may be prevented by administration of magnesium oxide tablets. Our findings provided new evidence for the management of serum magnesium levels in patients receiving cetuximab.

\section{Introduction}

Cetuximab is a monoclonal antibody that targets epidermal growth factor (EGFR) [1] and is used for the treatment of unresectable advanced or recurrent colorectal cancer, and head and neck cancer. Cetuximab causes hypomagnesemia because of suppression of magnesium reabsorption mediated by EGFRdependent transient receptor potential member 6 (TRPM6) [2]. The percentages of hypomagnesemia, hypokalemia, and hypocalcemia in patients receiving cetuximab were $34.9 \%, 8.0-12.6 \%$, and $16.8 \%$, respectively $[3,4]$. The median number of administrations at the onset of hypomagnesemia was 7 and 13 $[5,6]$; however, this remains controversial. Previous studies have focused on the incidence and related factors of cetuximab-induced hypomagnesemia. However, little is known about the relationship between the onset of hypomagnesemia and patient background, including electrolyte abnormalities before cetuximab administration and time-dependent changes of the serum magnesium levels. These findings are needed to appropriately monitor serum electrolyte levels after cetuximab administration.

Magnesium is an essential element, the fourth most abundant cation in humans, and plays important roles in many biological processes. The United States Food and Nutrition Board recommends a daily 
magnesium intake of $420 \mathrm{mg}$ for males and $320 \mathrm{mg}$ for females [7]. Magnesium absorption (30-50\%) occurs in the small intestine [7]. Most of the filtered magnesium in the kidney is reabsorbed in the thick ascending limb of Henle (60\%) [8]. Fine control of magnesium is performed in the distal tubule [9], and reabsorption in the kidney is assumed to be important for magnesium balance in the body.

Hypomagnesemia is generally defined as serum magnesium levels $<1.8 \mathrm{mg} / \mathrm{dL}$. Mild hypomagnesemia is often symptomless, but severe hypomagnesemia may induce fatal complications, such as cardiac arrhythmias if not treated [10]. A previous study reported that severe hypomagnesemia is difficult to treat by magnesium supplementation either orally or via injection [11]. It is important to prevent severe hypomagnesemia by monitoring serum magnesium levels over time. Magnesium is associated with other electrolytes, including sodium, potassium, and calcium. Magnesium is extruded into the blood compartment via a sodium-magnesium exchanger [12]. In the kidney, magnesium deficiency inhibits the renal outer medullary potassium channel and may cause hypokalemia [13]. Low intracellular magnesium causes hypocalcemia because of being impaired parathyroid hormone secretion $[14,15]$.

Reportedly, significant correlations were observed between potassium, magnesium, and calcium in the non-hypomagnesemia group receiving cetuximab, whereas not observed in the hypomagnesemia group [16]. We hypothesized that the onset of cetuximab-induced hypomagnesemia is associated with the other serum electrolyte levels before administration. Therefore, the relationships between serum magnesium levels and baseline serum electrolyte levels such as sodium, potassium, calcium, and magnesium were investigated. This study aims to provide new information regarding serum magnesium levels in patients receiving cetuximab and examine how patient backgrounds influence the onset of hypomagnesemia and time-dependent changes in serum magnesium levels.

\section{Materials And Methods}

\subsection{Patients and study design}

A retrospective study was performed to investigate serum magnesium levels in patients who received cetuximab at Kindai University Nara Hospital. Clinical data for patients with unresectable advanced or recurrent colorectal cancer or head and neck cancer treated with cetuximab from March 2010 to September 2020 were retrieved from the medical records. The main eligibility criteria included measurement of serum electrolyte levels, $>1$ cetuximab administration, and completion of the regimen. Patient serum creatinine (Scr), sodium, potassium, magnesium, and calcium levels were investigated before and after cetuximab administration. The serum calcium level was corrected using Payne's equation [17]. Creatinine clearance (Ccr) was calculated using the Cockcroft-Gault formula [18].

\subsection{Definition of electrolyte abnormalities}

Electrolyte abnormalities were graded according to the Common Terminology Criteria for Adverse Events version 5.0, translated by the Japanese Clinical Oncology Group (CTCAE ver. 5.0 JCOG version) [19]. Electrolyte abnormalities during cetuximab administration were defined as follows: hyponatremia, serum 
sodium level < $138.0 \mathrm{mmol} / \mathrm{L}$; hypokalemia, serum potassium level < $3.6 \mathrm{mmol} / \mathrm{L}$; hypomagnesemia, serum magnesium level $<1.8 \mathrm{mg} / \mathrm{dL}$; hypocalcemia, serum corrected calcium level $<8.8 \mathrm{mg} / \mathrm{dL}$.

\subsection{Statistical analysis}

Statistical analyses were performed using JMP Pro, version 15.0.0 (SAS Institute Inc., Cary, NC, USA). A pvalue less than 0.05 was considered significant. The relationship between the onset of hypomagnesemia and patient characteristics was evaluated using the likelihood ratio test and univariate logistic regression analysis. The multivariate logistic regression analysis was performed with the variables which had a pvalue $<0.05$ in the univariate logistic regression analysis. Furthermore, the area under the receiver operating characteristic (ROC) curve (AUC) was calculated to assess the performance of these logistic models. Serum magnesium levels were compared with the initial baseline by using a repeated-measures analysis of variance (ANOVA) with Tukey's test. To reveal changes in serum magnesium levels in patients with hypomagnesemia during the study period, serum magnesium levels were compared with and without hypomagnesemia by using a repeated-measures ANOVA between the two groups with regard to the number of administrations. Similarly, serum magnesium levels were compared with and without patient characteristics using a repeated-measures ANOVA.

\subsection{Ethical consideration}

This study was approved by the Ethics Committee of Kindai University Nara Hospital (approval ID: 1944) on April 20, 2020. All procedures in this study involving human participants were conducted following the ethical standards of the institutional research committee and the 1964 Declaration of Helsinki and its later amendments, or comparable ethical standards. Patients were not required to provide informed consent for study participation because this study was a retrospective study. We applied the opt-out method to obtain consent for this study. The opt-out method was approved by the Ethics Committee of Kindai University Nara Hospital.

\section{Results}

\subsection{Patient characteristics and treatment details}

Fig. 1 shows a flowchart of the patient selection procedure. A total of 113 patients received cetuximab during the study period. Forty-eight patients whose serum magnesium, calcium, or albumin levels were not measured during cetuximab treatment were excluded. Additionally, we excluded six patients who had only one administration of cetuximab and seven patients whose regimen was not completed. Table 1 shows the patient backgrounds. We identified 52 patients (40 males and 12 females) who received cetuximab during the study period. The mean age of the patients was $67.9 \pm 10.3$ years. Cetuximab was administrated for the treatment of colorectal cancer and head and neck cancer in 11 and 41 patients, respectively. The proportion of patients who had a history of anti-cancer therapy before cetuximab administration was $53.8 \%(28 / 52)$. The mean laboratory data were within the normal range. Table 2 shows the patient treatment details. The average treatment period was $50.5 \pm 30.9 \mathrm{~d}$. Regarding the list of regimens, the proportion of patients who received monotherapy and combination therapy group was 
$67.3 \%$ (35/52) and 32.7\% (17/52), respectively. Patients were administered one of the following regimens: cetuximab monotherapy; $\mathrm{CDDP}+5-\mathrm{FU}+$ cetuximab, which included cisplatin, 5-fluorouracil, and cetuximab; PCE therapy, which included paclitaxel, carboplatin, and cetuximab; CPT-11+cetuximab, which included irinotecan and cetuximab; high-dose DTX+cetuximab, which included docetaxel and cetuximab; CBDCA+5-FU+cetuximab, which included carboplatin, 5-fluorouracil, and cetuximab; SOX+cetuximab therapy, which included S-1, oxaliplatin, and cetuximab; and sLV5FU2+cetuximab, which included levofolinate, 5-FU, and cetuximab. Platinum-containing drugs were administered to 12 patients and eight patients received $\mathrm{CDDP}+5-\mathrm{FU}+$ cetuximab, which were the most frequent regimens. The proportion of patients who additionally received $\mathrm{MgO}$ tablet and $\mathrm{MgSO}_{4}$ injection was $57.7 \%$ (30/52) and 15.4\% (8/52), respectively.

\subsection{Effect of patient factors on the serum magnesium levels in patients}

Table 3 shows relationships between the onset of hypomagnesemia and patient characteristics during cetuximab administration. The onset of hypomagnesemia was associated with the baseline serum sodium levels [odds ratio (OR): $0.741,95 \% \mathrm{Cl}: 0.588-0.934, \mathrm{p}=0.003$ ], and a combination of $\mathrm{MgO}$ tablet (OR: $0.997,95 \% \mathrm{Cl}: 0.995-0.999, p=0.002$ ). However, the onset of hypomagnesemia was not associated with the baseline serum potassium, magnesium, or calcium levels because these electrolyte levels in the most of patients were within the normal range. Furthermore, no significant difference was observed between the onset of hypomagnesemia and the combined of platinum-containing drugs (OR: $2.333,95 \%$ $\mathrm{Cl}: 0.624-8.719, \mathrm{p}=0.209$ ) and $\mathrm{MgSO}_{4}$ injection (OR: $3.974,95 \% \mathrm{Cl}: 0.826-19.123, \mathrm{p}=0.079$ ). Table 4 shows the multivariable logistic regression analysis of relationships between the onset of hypomagnesemia and patient characteristics or treatment details. The multivariate logistic regression analysis revealed that the baseline serum sodium levels (OR: $0.729,95 \% \mathrm{Cl}: 0.552-0.963, \mathrm{p}=0.017$ ) and the combination of magnesium oxide tablet (OR: 0.997, 95\% Cl: $0.995-0.999, p=0.026$ ) were one of the independent factors for hypomagnesemia, and the model of $p$-value was $<0.001$. Additionally, the ROC curves of these multiple models were analyzed to assess the predictive abilities. Fig. 2 shows the ROC curves of patient characteristics and treatment details to detect the onset of hypomagnesemia. The predictive ability of a ROC curve may be assessed as follows: $0.50-0.70$, low; $0.70-0.90$, moderate; and $0.90-1.00$, high. The AUC value of the model was 0.801 , indicating fair predictive performances.

Fig. 3 shows the effects of cetuximab administration on the serum magnesium level in all patients, patients with hypomagnesemia among study period, patients administered $\mathrm{MgO}$ tablet, and patients with pre-hyponatremia. Serum magnesium levels tended to decline with an increasing number of administrations. Serum magnesium levels were significantly reduced in the hypomagnesemia group than in the non-hypomagnesemia group (least-square (LS) mean, 95\% confidence interval (Cl): $1.7 \mathrm{mg} / \mathrm{dL}$, $1.6-1.8 \mathrm{mg} / \mathrm{dL}$ vs. $2.1 \mathrm{mg} / \mathrm{dL}, 2.1-2.2 \mathrm{mg} / \mathrm{dL}, \mathrm{p}<0.001)$. The serum magnesium level before administration was not significantly different between the hypomagnesemia group and the nonhypomagnesemia group (LS mean, 95\% Cl: $2.0 \mathrm{mg} / \mathrm{dL}, 1.9-2.2 \mathrm{mg} / \mathrm{dL}$ vs. $2.2 \mathrm{mg} / \mathrm{dL}, 2.1-2.3 \mathrm{mg} / \mathrm{dL}$ ). However, the serum magnesium level after the two administrations was significantly different between the two groups (LS mean, 95\% Cl: $1.8 \mathrm{mg} / \mathrm{dL}, 1.7-1.9 \mathrm{mg} / \mathrm{dL}$ vs. $2.2 \mathrm{mg} / \mathrm{dL}, 2.1-2.3 \mathrm{mg} / \mathrm{dL}$ ). The serum 
magnesium level was significantly lower in the patient who had pre-hyponatremia (LS mean, 95\% Cl: 1.8, 1.7-1.9 mg/dL vs. 2.1, 2.0-2.1 mg/dL, p = 0.003). In the patient who developed pre-hyponatremia, the LS mean of serum magnesium level was less than $1.8 \mathrm{mg} / \mathrm{dL}$ after the five administrations. Additionally, the serum magnesium levels were significantly higher in the patients receiving combined MgO tablets (LS mean, 95\% Cl: 2.0, 1.9-2.1 mg/dL vs. 1.9, 1.8-2.0 mg/dL, $\mathrm{p}=0.041)$.

\section{Discussion}

In this study, we showed that serum magnesium levels are reduced in a time-dependent manner, and the onset of hypomagnesemia is affected by patient characteristics, including pre-hyponatremia and the combination of $\mathrm{MgO}$ tabs. In our study, the onset of hypomagnesemia was associated with the baseline serum sodium levels, but not with the baseline serum potassium, magnesium, or calcium levels. Because the baseline serum potassium, magnesium, or calcium levels in the most of patients were within the normal range. In distal tubules, the expression of EGFR-dependent TRPM6 is the greatest, and TRPM6 initiates fine control of magnesium excretion [20]. Reportedly, cetuximab inhibits TRPM6 in the distal tubules, which may result in magnesium deficiency in patients receiving cetuximab [2]. Franken et al. [12] reported that magnesium is reabsorbed into the cell by TRPM6 and is extruded into the blood compartment via a sodium-magnesium exchanger (SLC41A1) in exchange for sodium. Therefore, it is assumed that the serum magnesium level in patients was associated with the baseline serum sodium levels in our study. Although the onset of hypomagnesemia was not significantly associated with the combination of platinum-containing drugs, serum magnesium levels trended to be lower in patients who received platinum-containing drugs. Reportedly, cisplatin reduces mRNA expression of TRPM6 in rat kidneys [21] and induces the onset of hypomagnesemia [5, 22]. However, Stintzing et al. [23] reported that a significant difference was not observed between platinum and non-platinum receiving patients in terms of the course of serum magnesium levels. Furthermore, Tanaka et al. [24] reported that the median time until onset of hypomagnesemia was $72 d(11-393 d)$ with cetuximab and 123 d (31-218 d) with cisplatin. The treatment period in our study was $50.5 \mathrm{~d}$, which was shorter than that of Tanaka's study. Therefore, this may be why a difference in the incidence of hypomagnesemia during the treatment period was not observed between platinum and non-platinum receiving patients in our study. Nevertheless, it is important for patients who are treated with platinum-containing drugs to monitor serum magnesium levels because cisplatin affects the onset of hypomagnesemia. In summary, our results suggest that patients with hyponatremia before cetuximab administration should be carefully monitored for serum magnesium levels, and early magnesium supplementation may be needed to maintain serum electrolyte levels.

Serum magnesium levels were significantly reduced after five administrations, and the median day to the onset of hypomagnesemia was $22 \mathrm{~d}$. Magnesium in the body is stored in bone and muscle, and serum magnesium levels reflect only $1 \%$ of the body's magnesium content [25]. Hence, magnesium deficiencies in the body may be caused even if the serum magnesium levels are within the normal range. In our study, despite the serum magnesium levels being within the normal range, the body magnesium may be deficient after two administrations of cetuximab. Serum magnesium levels in patients receiving 
cetuximab were significantly reduced in the hypomagnesemia group compared with that of the nonhypomagnesemia group. A previous study necessitates treatment of hypomagnesemia when the patient displayed the presence of clinical symptoms and/or severe hypomagnesemia $(<1.25 \mathrm{mg} / \mathrm{dL})$ [9]. Fakih et al. [11] reported that patients with grade $3 / 4$ hypomagnesemia did not achieve their target serum magnesium level despite multiple magnesium infusions a week. These previous studies have shown that correction of serum magnesium levels is needed before severe hypomagnesemia occurs. Mild hypomagnesemia with no or only mild symptoms can be treated with oral magnesium supplementation $( \pm 360 \mathrm{mg} / \mathrm{d})[7,26]$. Our results suggest that patients who received cetuximab require oral magnesium supplementation when the serum magnesium level was less than $1.8 \mathrm{mg} / \mathrm{dL}$. The bioavailability of $\mathrm{MgO}$ is $4 \%$, which is the lowest of all magnesium supplements [9]. Administration of $\mathrm{MgO}$ tab up to $400 \mathrm{mg}$ three times a day was ineffective for grade $3 / 4$ hypomagnesemia [11]. However, in our study, the serum magnesium level was higher in the group with a combined $\mathrm{MgO}$ tab than in the control group. Zarif et al. [27] reported that continuous administration of the $\mathrm{MgO}$ tab according to the cisplatin dose reduces the decline in serum magnesium levels and the incidence of hypomagnesemia in cancer patients. Although further investigation is needed, the concomitant use of $\mathrm{MgO}$ tabs may contribute to delaying the onset of hypomagnesemia in patients receiving cetuximab. Furthermore, no significant difference was observed between the onset of hypomagnesemia and the combined $\mathrm{MgSO}_{4}$ injection. This result suggests that the incidence of hypomagnesemia was high in the patients who received $\mathrm{MgSO}_{4}$ injections because $\mathrm{MgSO}_{4}$ injections were administered after the onset of hypomagnesemia.

Notably, our study has several potential limitations. First, this study had a retrospective design, and the number of patients with electrolyte abnormalities was small. Second, our study period was short compared with previous studies. In the future, a prospective study is needed to evaluate the management of serum electrolyte levels using our findings. To our knowledge, this is the first study to show relationships between the onset of hypomagnesemia and patient factors including baseline serum sodium levels and the combination of $\mathrm{MgO}$ tabs, and between serum magnesium levels and the number of cetuximab administrations. Here, we provide new evidence that adequate management of serum electrolyte levels is necessary for maintaining chemotherapy.

\section{Conclusions}

Our study demonstrated that the onset of hypomagnesemia was associated with the combination of $\mathrm{MgO}$ tabs and the baseline serum sodium levels. Serum magnesium levels were reduced earlier in patients with pre-hyponatremia than in patients with non-pre-hyponatremia. Additionally, the incidence of hypomagnesemia was low in patients that received $\mathrm{MgO}$ tabs. After the second administrations of cetuximab, the serum magnesium level was significantly lower in the hypomagnesemia group than in the non-hypomagnesemia group. These findings suggest that baseline serum sodium levels before the administration can be an indicator of the serum magnesium level after administration of cetuximab, and two administrations of cetuximab may cause magnesium deficiency in the body even if the serum level is within the normal range. 


\section{Declarations}

Ethics approval and consent to participate: This study was approved by the Ethics Committee of Kindai University Nara Hospital (approval ID: 19-44) on April 20, 2020. All procedures in this study involving human participants were conducted following the ethical standards of the institutional research committee and the 1964 Declaration of Helsinki and its later amendments, or comparable ethical standards. Patients were not required to provide informed consent for study participation because this study was a retrospective study. We applied the opt-out method to obtain consent for this study. The optout method was approved by the Ethics Committee of Kindai University Nara Hospital.

Consent for publication: Not applicable.

Competing interests: The authors declare that they have no competing interests.

Funding: This research received no specific grant from any funding agency in the public, commercial, or not-for-profit sectors.

Data availability statement: The datasets generated and/or analysed during the current study are not publicly available due to the information could compromise the privacy of research participants but are available from the corresponding author on reasonable request.

Authors' contributions: KY analyzed the study data and wrote the manuscript with the assistance of NK, $\mathrm{AH}$, and $\mathrm{RO}$. $\mathrm{KY}, \mathrm{AH}$, and $\mathrm{RO}$ conceived the study design. $\mathrm{AH}, \mathrm{RO}, \mathrm{FO}, \mathrm{TN}$, and NK reviewed the manuscript. All authors have read and approved the final version of the manuscript.

Acknowledgements: Not applicable

\section{Authors' information:}

1. Kaito Yamashiro ${ }^{1,2}$ (kit9494ku@gmail.com, ORCID: 0000-0002-7785-1490)

2. Atsushi Hirata ${ }^{2}$ (hirata1976@med.kindai.ac.jp, ORCID: 0000-0002-4865-3807)

3. Ryosuke Ota ${ }^{2}$ (oota-ryousuke@med.kindai.ac.jp, ORCID: 0000-0003-0161-6766)

4. Fumihiko Ogata ${ }^{1}$ (ogata@phar.kindai.ac.jp, ORCID: 0000-0002-2755-8341)

5. Takehiro Nakamura ${ }^{1}$ (nakamura@phar.kindai.ac.jp, ORCID: 0000-0003-3548-2890)

6. Naohito Kawasaki ${ }^{1}$ (kawasaki@phar.kindai.ac.jp, ORCID: 0000-0002-9412-0868)

${ }^{1}$ Faculty of Pharmacy, Kindai University, 3-4-1, Kowakae, Higashi-Osaka, Osaka, 577-8502, Japan

${ }^{2}$ Department of Pharmacy, Kindai University Nara Hospital, 1248-1, Otodacho, Ikoma, Nara, 630-0293, Japan

English checker. Editage (www.editage.com) 


\section{References}

1. Ciardiello F, Tortora G. Anti-epidermal growth factor receptor drugs in cancer therapy. Expert Opin Investig Drugs. 2002;11(6):755-68.

2. Groenestege WMT, Thébault S, van der Wijst J, et al. Impaired basolateral sorting of pro-EGF causes isolated recessive renal hypomagnesemia. J Clin Invest. 2007;117(8):2260-7.

3. Cao Y, Liu L, Liao C, Tan A, Gao F. Meta-analysis of incidence and risk of hypokalemia with cetuximab-based therapy for advanced cancer. Cancer Chemother Pharmacol. 2010;66(1):37-42.

4. Wang Q, Qi Y, Zhang D, et al. Electrolyte disorders assessment in solid tumor patients treated with anti-EGFR monoclonal antibodies: a pooled analysis of 25 randomized clinical trials. Tumour Biol. 2015;36(5):3471-82.

5. Tano T, Une H, Fukui N, Nakajima Y, Kimura Y, Ueno T. Analysis of the incidence of cetuximabinduced hypomagnesemia in patients with oral cancer. Jpn J Oral Maxillofac Surg. 2018;64(11):634-9.

6. Nakamoto $E$, Kawakami $K$, Imada $H$, et al. Retrospective investigation on cetuximab-induced hypomagnesemia; Incidence, time of onset, and management. Jpn J Pharm Health Care Sci. 2011;37(7):403-9.

7. Baaij JHFd, Hoenderop JGJ, Bindels RJM. Magnesium in man: Implications for health and disease. Physiol Rev. 2015;95(1):1-46.

8. Curry JN, Yu ASL. Magnesium handling in the kidney. Adv Chronic Kidney Dis. 2018;25(3):236-43.

9. Van Laecke S. Hypomagnesemia and hypermagnesemia. Acta Clin Belg. 2019;74(1):41-7.

10. Soliman HM, Mercan D, Lobo SS, Mélot C, Vincent JL. Development of ionized hypomagnesemia is associated with higher mortality rates. Crit Care Med. 2003;31(4):1082-7.

11. Fakih MG, Wilding G, Lombardo J. Cetuximab-induced hypomagnesemia in patients with colorectal cancer. Clin Colorectal Cancer. 2006;6(2):152-6.

12. Franken GA, Adella A, Bindels RJ, de Baaij JH. Mechanisms coupling sodium and magnesium reabsorption in the distal convoluted tubule of the kidney. Acta Physiol. 2021;231:e13528.

13. Huang C-L, Kuo E. Mechanism of hypokalemia in magnesium deficiency. J Am Soc Nephrol. 2007;18(10):2649-52.

14. Tsujii T, Ogaki T, Nakae K, et al. Correlation between blood magnesium and calcium concentration in patients treated with an anti-EGFR antibody. J Pharm Health Care Sci. 2016;2:23.

15. Fatemi S, Ryzen E, Flores J, Endres DB, Rude RK. Effect of experimental human magnesium depletion on parathyroid hormone secretion and 1, 25-dihydroxyvitamin D metabolism. J Clin Endocrinol Metab. 1991;73(5):1067-72.

16. Yamashiro K, Hirata A, Ota R, Ogata F, Nakamura T, Kawasaki N. Relationship Between Serum Potassium, Magnesium, and Calcium in Patients Receiving Cetuximab Therapy. BPB Reports. 2021;4(1):22-6. 
17. Payne RB, Little AJ, Williams RB, Milner JR. Interpretation of serum calcium in patients with abnormal serum proteins. Br Med J. 1973;4(5893):643-6.

18. Cockcroft DW, Gault MH. Prediction of creatinine clearance from serum creatinine. Nephron. 1976;16(1):31-41.

19. Japanese Clinical Oncology Group. Common Terminology Criteria for Adverse Events version 5.0 Japanese Clinical Oncology Group. Available from:

http://www.jcog.jp/doctor/tool/CTCAE_v50_unyou_20190906.pdf. Accessed 27 April 2020.

20. Hoenderop JG, Bindels RJ. Epithelial $\mathrm{Ca}^{2+}$ and $\mathrm{Mg}^{2+}$ channels in health and disease. J Am Soc Nephrol. 2005;16(1):15-26.

21. Ledeganck KJ, Boulet GA, Bogers JJ, Verpooten GA, De Winter BY. The TRPM6/EGF pathway is downregulated in a rat model of cisplatin nephrotoxicity. PLoS One. 2013;8(2):e57016.

22. Inose R, Takahashi K, Nishikawa T, Nagayama K. Analysis of factors influencing the development of hypomagnesemia in patients receiving cetuximab therapy for head and neck cancer. Yakugaku Zasshi. 2015;135(12):1403-7.

23. Stintzing $S$, Fischhaber $D$, Mook $C$, et al. Clinical relevance and utility of cetuximab-related changes in magnesium and calcium serum levels. Anticancer Drugs. 2013;24(9):969-74.

24. Tanaka H, Yoshiba $Y$, Watanabe T, Satoh M, Ishii T. Analysis of patients with hypomagnesemia using the Japanese Adverse Drug Event Report database (JADER). J Pharm Pharm Sci. 2018;21(1):46-53.

25. Elin RJ. Magnesium: The fifth but forgotten electrolyte. Am J Clin Pathol. 1994;102(5):616-22.

26. Yamamoto $M$, Yamaguchi T. Causes and treatment of hypomagnesemia. Clin Calcium. 2007;17(8):1241-8.

27. Zarif Yeganeh M, Vakili M, Shahriari-Ahmadi A, Nojomi M. Effect of oral magnesium oxide supplementation on cisplatin-induced hypomagnesemia in cancer patients: A randomized controlled trial. Iran J Public Health. 2016;45(1):54-62.

\section{Tables}




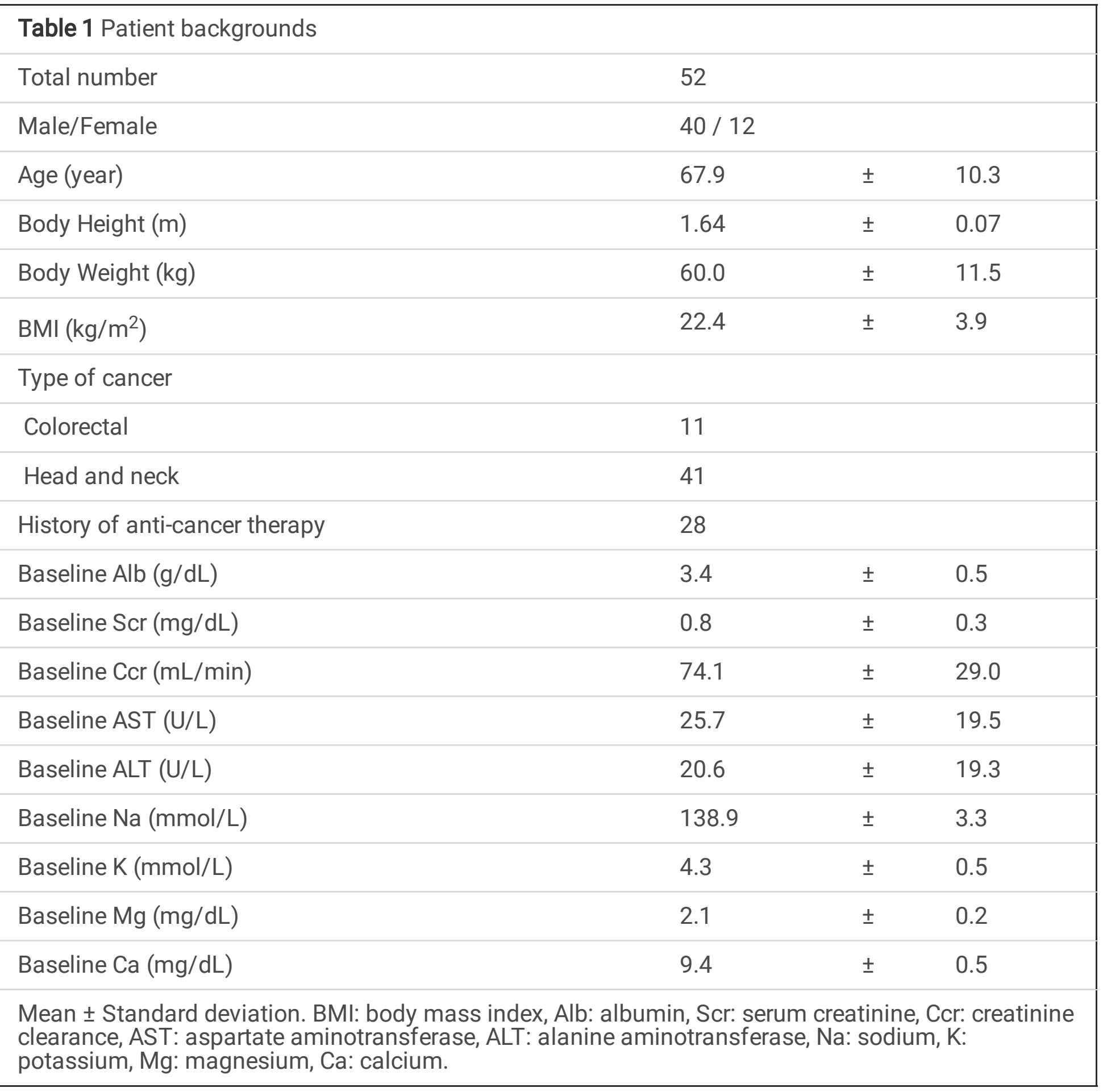


Table 2 Patient treatment details

\begin{tabular}{ll} 
Treatment period (d) & $50.5 \pm 30.9$ \\
\hline List of regimens & 35 \\
\hline Monotherapy (cetuximab only) & 17 \\
\hline Combination therapy & 8 \\
\hline CDDP+5-FU+cetuximab & 2 \\
\hline PCE & 2 \\
\hline CPT-11+cetuximab & 2 \\
\hline High dose DTX+cetuximab & 1 \\
\hline CBDCA+5-FU+cetuximab & 1 \\
\hline SOX+cetuximab & 1 \\
\hline SLV5FU2+cetuximab & \\
\hline Combined therapy & 41 \\
\hline Radiation therapy & 12 \\
\hline Platinum-containing drugs & 30 \\
\hline MgO tablet & 8 \\
\hline MgSO 4 injection & \\
\hline $\begin{array}{l}\text { Mean } \pm \text { standard deviation. CDDP: cisplatin, 5-FU: 5-fluorouracil, PCE: paclitaxel+ } \\
\text { sLV5FU2: levofolinate+5-FU. }\end{array}$ & \\
\hline
\end{tabular}


Table 3 Relationships between hypomagnesemia and patient characteristics

$\begin{array}{llll}\text { Patient backgrounds and treatment details } & \text { Odds ratio } & 95 \% \mathrm{Cl} & \text { p-value }\end{array}$

Patient backgrounds

\begin{tabular}{llll} 
Male / Female a) & 3.333 & $0.643-17.265$ & 0.119 \\
\hline Age (year) & 0.952 & $0.899-1.008$ & 0.080 \\
\hline BMI $\left(\mathrm{kg} / \mathrm{m}^{2}\right)$ & 0.960 & $0.825-1.117$ & 0.466
\end{tabular}

Type of cancer

\begin{tabular}{|c|c|c|c|}
\hline Colorectal / Head and neck a) & 1.102 & $0.275-4.415$ & 0.891 \\
\hline History of anti-cancer therapy a) & 1.571 & $0.492-5.022$ & 0.443 \\
\hline Baseline Alb (g/dL) & 0.758 & $0.314-1.832$ & 0.539 \\
\hline Baseline Scr (mg/dL) & 0.652 & $0.069-6.114$ & 0.707 \\
\hline Baseline $\mathrm{Ccr}(\mathrm{mL} / \mathrm{min})$ & 1.010 & $0.991-1.031$ & 0.299 \\
\hline Baseline AST (U/L) & 1.022 & $0.982-1.065$ & 0.190 \\
\hline Baseline ALT (U/L) & 0.989 & $0.954-1.026$ & 0.538 \\
\hline Baseline $\mathrm{Na}(\mathrm{mmol} / \mathrm{L})$ & 0.741 & $0.588-0.934$ & $0.003^{* *}$ \\
\hline Baseline K (mmol/L) & 2.180 & $0.584-8.136$ & 0.237 \\
\hline Baseline Mg (mg/dL) & 0.127 & $0.003-5.528$ & 0.268 \\
\hline Baseline $\mathrm{Ca}(\mathrm{mg} / \mathrm{dL})$ & 3.103 & $0.844-11.403$ & 0.077 \\
\hline \multicolumn{4}{|l|}{ Treatment details } \\
\hline Monotherapy / Combination a) & 0.308 & $0.091-1.039$ & 0.055 \\
\hline Radiation therapy a) & 0.907 & $0.227-3.635$ & 0.891 \\
\hline Platinum-containing drugs a) & 2.333 & $0.624-8.719$ & 0.209 \\
\hline MgO tablet (mg/d) & 0.997 & $0.995-0.999$ & $0.002^{\star \star}$ \\
\hline $\mathrm{MgSO}_{4}$ injection ${ }^{\mathrm{a})}$ & 3.974 & $0.826-19.123$ & 0.079 \\
\hline Treatment period $(\mathrm{d})$ & 1.010 & $0.992-1.029$ & 0.277 \\
\hline
\end{tabular}

Cl: confidence interval, BMI: body mass index, Alb: albumin, Scr: serum creatinine, Ccr: creatinine clearance, AST: aspartate aminotransferase, ALT: alanine aminotransferase, Na: sodium, K: potassium, Mg: magnesium, Ca: calcium, MgO: magnesium oxide, MgSO4: magnesium sulfate. ${ }^{\star \star} \mathrm{p}<$ 0.01 , p: statistical significance obtained using the univariate logistic regression analysis, and ${ }^{a)}$ the likelihood ratio test. 
Table 4 Multivariate logistic regression analysis of hypomagnesemia

\begin{tabular}{llll} 
& Odds ratio & $95 \% \mathrm{Cl}$ & \multicolumn{2}{c}{ p-value } \\
Baseline $\mathrm{Na}(\mathrm{mmol} / \mathrm{L})$ & 0.729 & $0.552-0.963$ & $0.026^{*}$ \\
MgO tablet $(\mathrm{mg} / \mathrm{d})$ & 0.997 & $0.995-0.999$ & $0.017^{*}$ \\
p-value & $<0.001^{* \star *}$ & & \\
AUC & 0.801 & &
\end{tabular}

Cl: confidence interval, Na: sodium, MgO: magnesium oxide. ${ }^{* \star *} \mathrm{p}<0.001,{ }^{*} \mathrm{p}<0.05, \mathrm{p}$ : statistical significance obtained using the multivariate logistic regression analysis.

Figures

Patients who received cetuximab $(\mathrm{n}=113)$

- Patients whose serum magnesium levels were not measured $(n=46)$

- Patients whose serum calcium levels were not measured $(n=1)$

- Patients who had only one administration $(n=6)$

- Patients whose serum albumin levels were not measured $(n=1)$

- Patients whose regimen was not completed $(\mathrm{n}=7)$

Study patients $(\mathrm{n}=52)$

\section{Figure 1}

Flowchart of the patient selection 


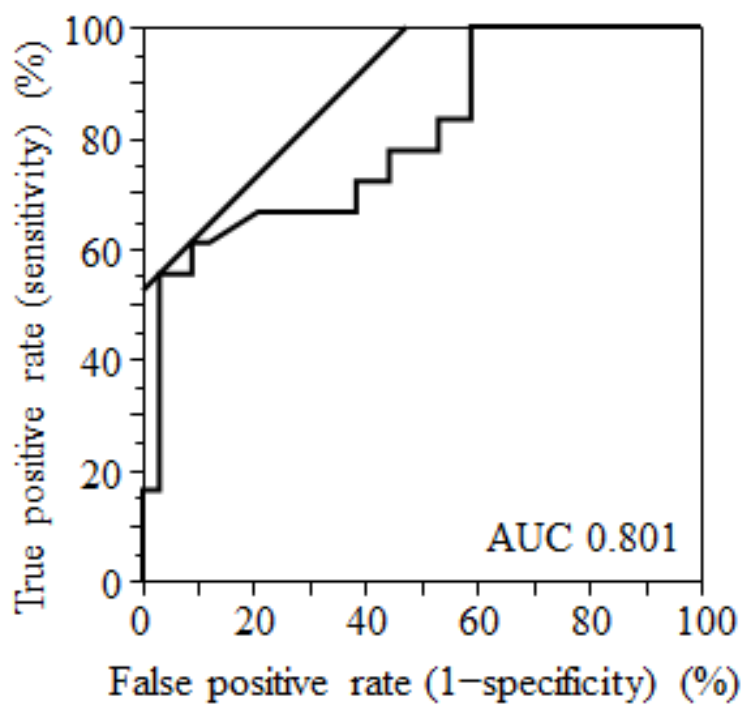

Figure 2

Receiver operating characteristic curves of patient characteristics and treatment details to detect hypomagnesemia. The patient characteristics were baseline serum sodium levels $(\mathrm{mmol} / \mathrm{L})$ and a combination of $\mathrm{MgO}$ tablets $(\mathrm{mg} / \mathrm{d})$. 


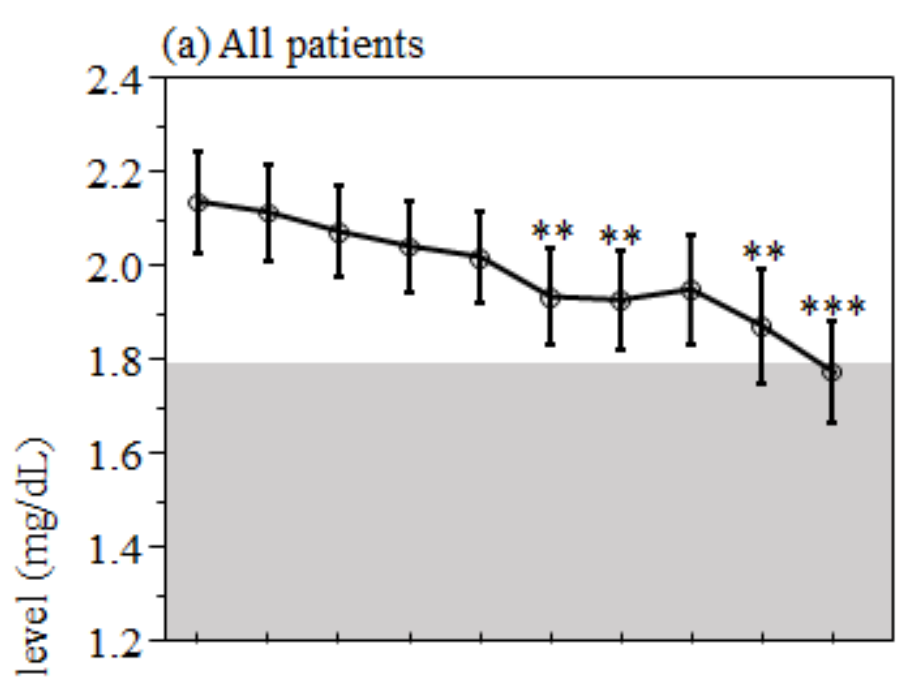

(b) Hypomagnesemia among study period

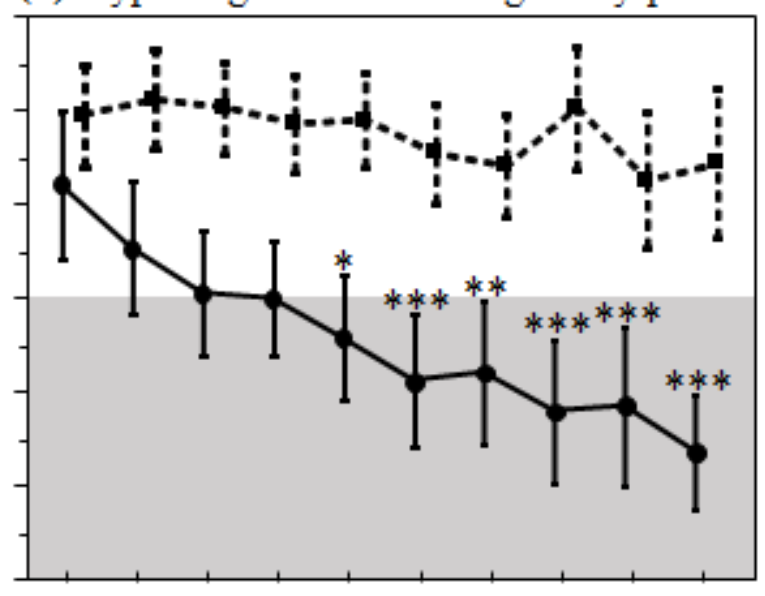

点

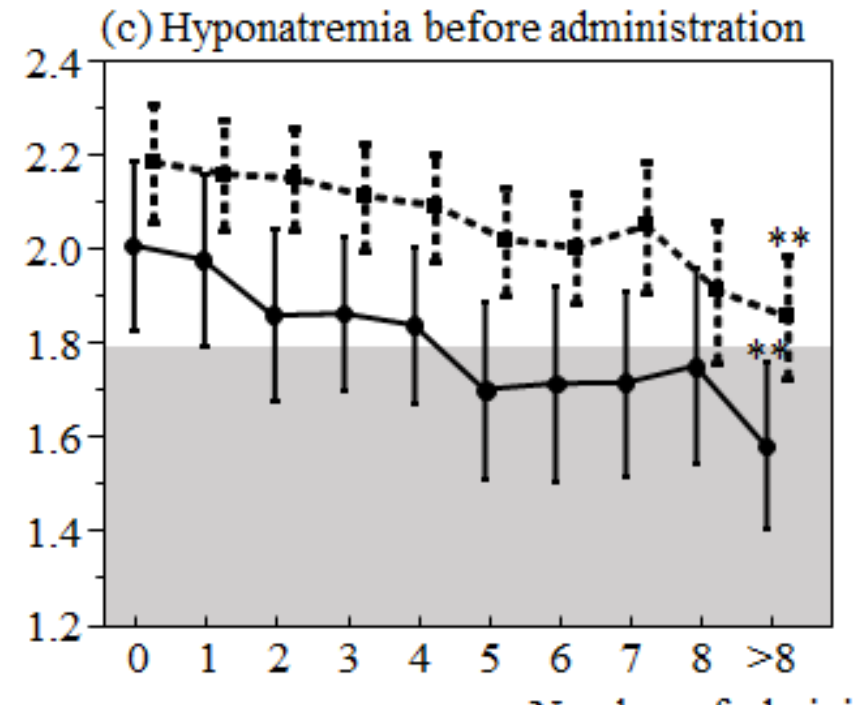

(d) Combination of $\mathrm{MgO}$ tablet

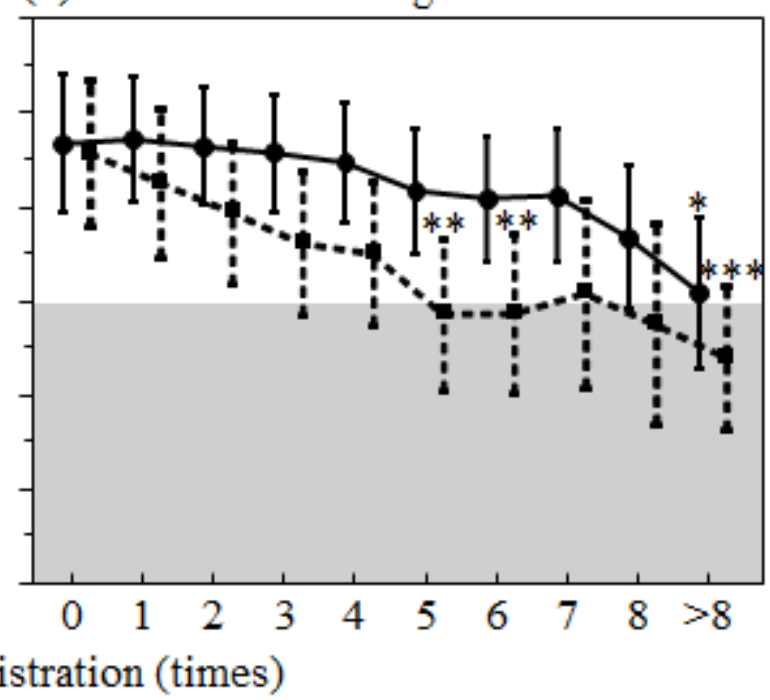

$\ominus$ : All patients, $-\bullet-$ Yes, $-\mathbf{- 1}:$ No, $\square:$ Range of hypomagnesemia

Figure 3

Effects of cetuximab administration on the serum magnesium level. The data are presented as the leastsquare mean $+95 \%$ confidence interval. p: statistical significance obtained using the repeated-measures ANOVA, ${ }^{*}<<0.05,{ }^{*} p<0.01, * \star * p<0.001, v s$. initial baseline. 